\title{
CAPTACIÓN DE CARBONO EN SUELOS ASOCIADOS A Pinus greggii Engelm. Y Pinus oaxacana Mirov. EN LA MIXTECA ALTA, OAXACA
}

\section{CARBON SEQUESTRATION IN SOILS ASSOCIATED WITH Pinus greggii Engelm. AND Pinus oaxacana Mirov. IN THE HIGH MIXTECA, OAXACA}

\author{
María López Ortiz ${ }^{1}$, Reyna Belén Sánchez García ${ }^{1}$, José Rafael Contreras Hinojosa², Adolfo Dagoberto Armenta \\ Bojórquez $^{3}$ y Jaime Alberto Félix Herrán ${ }^{4}$
}

\begin{abstract}
Resumen
Se determinó la cantidad de hojarasca acumulada, la materia orgánica (MO) y el Carbono Orgánico (CO) en suelos asociados a trece procedencias de Pinus greggii Engelm. y ocho procedencias de Pinus oaxacana Mirov. en Tlacotepec Plumas (TP) y Magdalena Zahuatlán (MZ), Oaxaca, México. El contenido de hojarasca se obtuvo en sitios de muestreo de $0.25 \mathrm{~m}^{2}$, recolectando todo el mantillo presente. En el mismo sitio de muestreo se abrió un perfil de $20 \mathrm{~cm}$ de profundidad, y se tomó una muestra de suelo, la cual se tamizó ( $<2 \mathrm{~mm}$ ) y se dejó secar por 24 h. Se le determinó el pH del extracto de pasta saturada y el \% de materia orgánica (MO); el \% de MO se multiplicó por 0.58 para convertirlo en Carbono Orgánico (CO). Para $P$. greggii, El Madroño acumuló más hojarasca en ambas comunidades (27.87 $\mathrm{Mg} \mathrm{ha}^{-1}$ y $21.85 \mathrm{Mg} \mathrm{ha}^{-1}$, respectivamente); en TP, Tres lagunas acumuló mayor contenido de MO y CO (7.65\% y 26.62\%, respectivamente); mientras que en MZ, Jamé fue la que presentó mayor MO y CO (7.91\% y 24.78\%, respectivamente); para $P$. oaxacana, Yudolahuerta presentó la mayor acumulación de hojarasca en TP (10.57 Mg ha $\left.{ }^{-1}\right)$, y en MZ fue Magdalena Zahuatlán (3.68 $\mathrm{Mg} \mathrm{ha}^{-1}$ ); mientras que el mayor contenido de MO se encontró en Magdalena Zahuatlán (6.99\%) en TP y Los Molinos presentó el mayor contenido de CO (9.74\%). Se presentó variación en cuanto a la concentración de CO entre localidades, especies y procedencias. Palabras clave: materia orgánica, sumidero de carbono, hojarasca, absorción de $\mathrm{CO}_{2}$.
\end{abstract}

\begin{abstract}
The amount of litter accumulated, organic matter (MO) and organic carbon (CO) in soils associated to thirteen provenances of Pinus greggii Engelm. and eight provenances of Pinus oaxacana Mirov. at Tlacotepec Plumas (TP) and Magdalena Zahuatlán (MZ), Oaxaca, México were determined. The amount of litter was obtained in a sampling site of $0.25 \mathrm{~m}^{2}$, gathering all the litter around. In the same sampling site, a profile of $20 \mathrm{~cm}$ of depth was opened in order to take a soil sample, which was sieved $(<2 \mathrm{~mm})$ and dried for $24 \mathrm{~h}$. Then the $\mathrm{pH}$ of the saturated paste extract and the $\%$ of organic matter (MO) were determined. The $\%$ of MO was multiplied by 0.58 to convert it into organic carbon (CO). For $P$. greggii, El Madroño accumulated more litter in both communities (27.87 $\mathrm{Mg} \mathrm{ha}^{-1}$ and 21.85 $\mathrm{Mg} \mathrm{ha}^{-1}$, respectively); at TP, Tres Lagunas accumulated the highest content of MO and CO (7.65\% and 26.62\%, respectively); while at MZ, Jamé showed higher amount of MO and CO (7.91\% and 24.78\%, respectively); For P. oaxacana, Yudolahuerta showed higher amount of litter at TP (10.57 $\left.\mathrm{Mg} \mathrm{ha}^{-1}\right)$, and at MZ it was Magdalena Zahuatlán (3.68 $\left.\mathrm{Mg} \mathrm{ha}^{-1}\right)$. The highest amount of MO was found at Magdalena Zahuatlán (6.99\%) in TP and Los Molinos showed the highest amount of $\mathrm{CO}(9.74 \%)$. Variation in the concentration of $\mathrm{CO}$ between localities, species and provenances was found.
\end{abstract}

Key words: organic matter, carbon sink, litter, $\mathrm{CO}_{2}$ absorption.

\section{Introducción.}

El clima de nuestro planeta lo determinan una serie de procesos físicos, químicos y biológicos que ocurren en la atmósfera, suelo y océano (Denman et al., 2007). Cambio climático se refiere a la variación en el estado del clima y dicho cambio puede persistir por un periodo prolongado, normalmente décadas; este cambio puede deberse a procesos naturales internos o externos, como son: modulación de ciclos solares; erupciones volcánicas; cambios antropogénicos persistentes en la composición atmosférica o cambios de uso del suelo (IPCC, 2014).

El cambio climático afecta a los bosques y viceversa, los dos están ligados; las variaciones en los patrones del clima (elevación de temperatura, alteración en los patrones de lluvia y otros eventos climáticos frecuentes y extremos) provocan estrés en el bosque; por otro lado, los bosques atrapan y almacenan 
$\mathrm{CO}_{2}$, teniendo un papel muy importante en la mitigación del cambio climático (Osman, 2013). La agricultura, silvicultura y otros usos del suelo (AFOLU, por sus siglas en inglés) son responsables de aproximadamente un cuarto ( 10-12 $\mathrm{GtCO}_{2}$ eq año-1) de las emisiones de Gases de Efecto Invernadero (GEI) antropogénico, principalmente por la deforestación y la emisiones agrícolas del ganado, suelo y manejo de nutrimentos (Smith et al., 2014).

El bosque desempeña un papel muy importante en el cambio climático y en las formas de mitigación del mismo, lo cual es un reto para el siglo XXI. Esto debido a que al reducir el potencial de mitigación del cambio climático de los bosques, se pierden muchos beneficios de los ecosistemas (Osman, 2013).

Las plantas durante la fotosíntesis, absorben el $\mathrm{CO}_{2}$ y lo convierten en $\mathrm{O}_{2}$, pero una parte del $\mathrm{C}$ queda inmovilizado en sus tejidos y otra parte regresa al suelo en forma de exudados. Por lo tanto, los bosques, que representan aproximadamente el 30\% de la superficie terrestre (FAO, 2006), almacenan cerca de 45\% del C en ecosistemas terrestres (Anderegg et al., 2012).

A nivel global la biomasa forestal almacena $283 \mathrm{Gt}$ de $\mathrm{C}$ en su biomasa, $38 \mathrm{Gt}$ en madera muerta y $317 \mathrm{Gt}$ en el suelo forestal (los primeros $30 \mathrm{~cm}$ ) y la hojarasca. El contenido total de $\mathrm{C}$ en los ecosistemas forestales se estimó en 638 Gt para el 2005, cifra mayor que la concentración de C en la atmósfera (Osman, 2013).

Price et al. (2012) sugieren que la naturaleza y condición de los bosques es importante para el secuestro de carbono en el suelo y los procesos de almacenamiento. Sin embargo la deforestación puede reducir la capacidad del suelo forestal de almacenar $\mathrm{CO}_{2}$ (Gorte \& Sheikh, 2010).

México es un país megadiverso, por su diversidad de bosques y selvas, los cuales por sus servicios ambientales, regulan el ciclo hidrológico y la captura de carbono, entre otros beneficios (SEMARNAT, 2007). Aun cuando es del dominio público la importancia de estos ecosistemas, la tasa de deforestación es de 155 mil ha al año en el periodo del 2005 al 2010 (SEGOB, 2014). La deforestación no solo implica la perdida de cobertura vegetal, sino también el impacto al suelo, y los ciclos biogeoquímicos que se llevan a cabo en él, como la captación del C por parte de la materia orgánica del suelo (Félix-Herrán et al., 2014).

Esta investigación pretende estimar la cantidad de carbono en suelos de plantaciones de Pinus greggii Engelm. y Pinus oaxacana Mirov. de diferentes procedencias, en dos comunidades de la región Mixteca del estado de Oaxaca, y así conocer si existe variación en cuanto a captura y almacenamiento de C en función de la especie.

\section{Materiales y métodos.}

Elección del sitio.

Las plantaciones de estudio se establecieron en 1997 en las localidades de Tlacotepec Plumas y Magdalena Zahuatlán, Oaxaca. Para determinar los sitios de muestreo se utilizó un muestreo simple aleatorio, tomando muestra de cuatro sitios por especie (Castillo, 2005).

Obtención de muestras de material vegetal (hojarasca).

Para determinar el contenido de hojarasca se delimitó un sitio de muestreo de $0.25 \mathrm{~m}^{2}$, dentro del cual se colectó todo el mantillo (ramillas y hojarasca), se clasificó como: materia orgánica que se podía identificar y materia orgánica humificada. Las muestras se dejaron secar a la sombra y después se determinó el peso seco, el cual se extrapoló a 1 ha.

Obtención de muestras de suelo.

En el cuadrante donde se tomó la muestra de mantillo, se abrió un perfil con una profundidad de 20 cm, para Pinus greggii se abrieron 52 perfiles (13 procedencias y 4 sitios de muestreo) y para Pinus oaxacana se abrieron 32 perfiles (8 procedencias y 4 sitios de muestreo). Las muestras se secaron a la

Tabla 1. Cantidad de acículas $\left(\mathrm{Mg} \mathrm{ha}^{-1}\right)$ (A) y carbono (Mg C ha-1) (CA) de $P$. greggii Engelm. acumuladas por procedencia en las dos comunidades.

\begin{tabular}{llllll}
\hline \multirow{2}{*}{ Procedencia } & \multicolumn{2}{c}{ Tlacotepec Plumas } & & \multicolumn{2}{c}{ Magdalena Zahuatlán } \\
\cline { 2 - 3 } \cline { 5 - 6 } & \multicolumn{1}{c}{$\mathrm{A}$} & \multicolumn{1}{c}{$\mathrm{CA}$} & & $\mathrm{A}$ & $\mathrm{CA}$ \\
\hline Xichicoatlán & $18.16 \mathrm{f} \pm 2.0$ & $10.53 \mathrm{f} \pm 0.8$ & & $8.21 \mathrm{~g} \pm 2.0$ & $4.76 \mathrm{~g} \pm 0.4$ \\
Puerto San Juan & $17.01 \mathrm{~g} \pm 1.6$ & $9.86 \mathrm{~g} \pm 1.0$ & & $7.25 \mathrm{i} \pm 1.3$ & $4.21 \mathrm{i} \pm 0.3$ \\
El Piñón & $19.06 \mathrm{e} \pm 0.6$ & $11.05 \mathrm{e} \pm 0.1$ & & $10.93 \mathrm{e} \pm 1.4$ & $6.34 \mathrm{e} \pm 0.2$ \\
Los Lirios & $12.13 \mathrm{j} \pm 1.0$ & $7.03 \mathrm{j} \pm 0.2$ & & $12.47 \mathrm{~d} \pm 1.3$ & $7.23 \mathrm{~d} \pm 0.1$ \\
Com. Durango & $22.18 \mathrm{~b} \pm 0.6$ & $12.86 \mathrm{~b} \pm 0.4$ & & $8.39 \mathrm{f} \pm 0.6$ & $4.86 \mathrm{f} \pm 0.6$ \\
Molango & $20.35 \mathrm{~d} \pm 1.4$ & $11.80 \mathrm{~d} \pm 0.7$ & & $20.53 \mathrm{~b} \pm 1.5$ & $11.91 \mathrm{~b} \pm 0.4$ \\
Pto. Los Conejos & $12.19 \mathrm{i} \pm 0.4$ & $7.07 \mathrm{i} \pm 0.2$ & & $1.83 \mathrm{~m} \pm 0.8$ & $1.06 \mathrm{~m} \pm 0.4$ \\
Laguna Atezca & $16.92 \mathrm{~h} \pm 0.8$ & $9.81 \mathrm{~h} \pm 0.2$ & & $7.48 \mathrm{~h} \pm 1.5$ & $4.34 \mathrm{~h} \pm 0.3$ \\
El Madroño & $27.87 \mathrm{a} \pm 1.7$ & $16.16 \mathrm{a} \pm 0.2$ & & $21.85 \mathrm{a} \pm 1.7$ & $12.67 \mathrm{a} \pm 0.3$ \\
Jamé & $11.43 \mathrm{k} \pm 1.3$ & $6.62 \mathrm{k} \pm 0.6$ & & $13.38 \mathrm{c} \pm 1.3$ & $7.76 \mathrm{c} \pm 0.4$ \\
Santa Anita & $11.08 \mathrm{l} \pm 1.0$ & $6.43 \mathrm{l} \pm 0.2$ & & $5.19 \mathrm{j} \pm 0.6$ & $3.01 \mathrm{j} \pm 0.4$ \\
Ej. 18 de marzo & $9.97 \mathrm{~m} \pm 1.2$ & $5.78 \mathrm{~m} \pm 0.1$ & & $5.07 \mathrm{k} \pm 1.0$ & $2.94 \mathrm{k} \pm 0.2$ \\
Tres lagunas & $21.38 \mathrm{c} \pm 1.3$ & $12.40 \mathrm{c} \pm 0.4$ & & $4.20 \mathrm{l} \pm 1.2$ & $2.43 \mathrm{l} \pm 0.3$ \\
\hline *Letras diferentes & entre columnas de la misma & procedencia indican diferencias \\
significativas entre el contenido de acículas, de acuerdo a la prueba de rangos múltiples \\
de Tukey (p<0.05). El dato después del \pm es el error estándar de la media.
\end{tabular}


sombra y se tamizaron (malla de $2 \mathrm{~mm}$ ). A las muestras se les determinó el $\mathrm{pH}$ del extracto de pasta saturada (Soil Survey Staff, 2014) y el carbono orgánico (López, 2012) presente, mismo que se extrapoló a una ha (Mg C ha-1).

Cuantificación del C orgánico de muestras de suelo.

El contenido de materia orgánica de las muestras de suelo se determinó por el método AS-07 (NOM-021RECNAT-2000), el cual se relacionó con el volumen de suelo contenido en $20 \mathrm{~cm}$ de profundidad por ha considerando una densidad aparente de $1.2 \mathrm{~g} \mathrm{~cm}^{-3}$ (2400 Mg de suelo ha ${ }^{-1}$ ) (López, 2012); para calcular el Carbono orgánico se multiplicó el porcentaje obtenido de la materia orgánica se multiplicó por 0.58, que es el factor de conversión de materia orgánica a carbono orgánico (Pribyl, 2010).

Análisis estadístico.

Se utilizó un diseño en bloques completos al azar para cada especie, siendo los bloques las comunidades Tlacotepec Plumas y Magdalena Zahuatlán, y las procedencias de cada especie los tratamientos, es decir, para Pinus greggii se tendrían 13 procedencias y para Pinus oaxacana se tendrían 8 procedencias, con 12 repeticiones cada una. El análisis de varianza (ANDEVA) se realizó en el paquete estadístico SAS versión 9.0 (2002), y para la comparación de medias se utilizó la prueba de rangos múltiples de Tukey a un nivel de significancia de 0.05 . Se comprobaron los supuestos básicos de normalidad con el estadístico de Shapiro-Wilk, y la homogeneidad de varianzas con la prueba de Brown y Forsythe.

\section{Resultados y discusión.}

Cantidad de hojarasca y $\mathrm{C}$ de la hojarasca.

Para ambas especies Pinus greggii Engelm. (Tabla 1) y Pinus oaxacana Mirov. (Tabla 2) se evaluó la cantidad de mantillo acumulada en el suelo, derivado de las ramillas y hojarasca, el monto obtenido se presenta como contenido de carbono ( $\left.\mathrm{Mg} \mathrm{C} \mathrm{ha}^{-1}\right)$.

Se encontró diferencia estadísticamente significativa $(\mathrm{p}<0.05)$ entre el carbono acumulado en los suelos asociados a $P$. greggii en las dos comunidades, como se puede observar en la Tabla 1 , también se puede apreciar que la cantidad de C acumulado en los suelos fue mayor en Tlacotepec Plumas que en Magdalena Zahuatlán. También se encontró que la procedencia $\mathrm{El} \mathrm{Madroño} \mathrm{fue} \mathrm{la} \mathrm{que}$ acumuló mayor cantidad de acículas y de $\mathrm{C}$ en ambas comunidades, que fue de 27.87 $\mathrm{Mg} \mathrm{C} \mathrm{ha}^{-1}$ y $16.16 \mathrm{Mg}$ $\mathrm{C}$ ha $^{-1}$, respectivamente para Tlacotepec Plumas y de 21.85 $\mathrm{Mg} \mathrm{C} \mathrm{ha}^{-1}$ y $12.67 \mathrm{Mg} \mathrm{C} \mathrm{ha}^{-1}$, respectivamente para Magdalena Zahuatlán.

En un estudio similar, Gutiérrez-Vázquez et al. (2012), evaluaron la caída de hojarasca (acículas) en suelos asociados a Pinus greggii y P. cembroides, encontraron que la caída de hojarasca fue mayor en $P$. greggii con $1.072 \mathrm{Mg} \mathrm{C} \mathrm{ha}^{-1}$ y en $P$. cembroides fue $0.976 \mathrm{Mg} \mathrm{C} \mathrm{ha-1}$. Los autores atribuyen esto a que la caída de hojarasca está en función de la especie, así como de factores climáticos (viento y temperatura) que influyen para obtener variaciones en cuanto a la cantidad de hojarasca acumulada.

En P. oaxacana Mirov. (Tabla 2) al realizar la comparación entre las comunidades, se encontró diferencia estadísticamente significativa $(\mathrm{p}<0.05)$ entre el contenido de carbono acumulado en el suelo asociado a la especie, se observó el mismo comportamiento que en $P$. greggii, se puede apreciar que el $\mathrm{C}$ acumulado fue mayor en Tlacotepec Plumas que en Magdalena Zahuatlán. También se encontró que la procedencia Yudolahuerta fue la que acumuló mayor cantidad de acículas y de Carbono en Tlacotepec Plumas con $10.57 \mathrm{Mg} \mathrm{C}$ ha $^{-1}$ y $6.13 \mathrm{Mg} \mathrm{C} \mathrm{ha-1}$ respectivamente; mientras que en Magdalena Zahuatlán fue Magdalena Zahuatlán con 3.68 Mg C ha${ }^{1}$ y $2.14 \mathrm{Mg} \mathrm{C}^{-1}$ respectivamente.

Comparando las dos especies, en Pinus greggii la cantidad de acículas y de carbono $\left(\mathrm{Mg} \mathrm{C} \mathrm{ha}^{-1}\right)$ fue tres veces mayor que en Pinus oaxacana en la Tlacotepec plumas y siete veces mayor que la encontrada en Magdalena Zahuatlán.

Cabe señalar que la caída de hojarasca en las dos especies estudiadas es menor a la reportada en otras especies del género Pinus, por ejemplo, Pérez et al. (2006) reportan una caída de $15.930 \mathrm{Mg} \mathrm{C} \mathrm{ha}^{-1}$ para $P$. taeda L.; en otro ensayo, Návar-Cháidez y Jurado-

Tabla 2. Cantidad de acículas ( $\left.\mathrm{Mg} \mathrm{ha}^{-1}\right)$ (A) y carbono (Mg C ha-1) (CA) de Pinus oaxacana Mirov. acumuladas por procedencia en las dos comunidades.

\begin{tabular}{llllcc}
\hline Procedencia & \multicolumn{2}{c}{ Tlacotepec Plumas } & & \multicolumn{2}{c}{ Magdalena Zahuatlán } \\
\cline { 2 - 3 } \cline { 6 - 7 } & \multicolumn{1}{c}{$\mathrm{A}$} & $\mathrm{C} \mathrm{A}$ & & $\mathrm{A}$ & $\mathrm{CA}$ \\
\hline Tlacotepec Plumas & $4.51 \mathrm{~d} \pm 1.4$ & $2.61 \mathrm{~d} \pm 0.2$ & & $2.66 \mathrm{~d} \pm 2.0$ & $1.54 \mathrm{~d} \pm 0.1$ \\
San Miguel Peras & $9.52 \mathrm{~b} \pm 0.7$ & $5.52 \mathrm{~b} \pm 0.8$ & & $3.58 \mathrm{~b} \pm 0.4$ & $2.07 \mathrm{~b} \pm 0.2$ \\
San Miguel Aloapan & $3.99 \mathrm{e} \pm 0.8$ & $2.31 \mathrm{e} \pm 0.3$ & & $2.61 \mathrm{e} \pm 1.4$ & $1.51 \mathrm{e} \pm 0.4$ \\
Magdalena Zahuatlán & $2.83 \mathrm{~h} \pm 0.9$ & $1.64 \mathrm{~h} \pm 0.2$ & & $3.68 \mathrm{a} \pm 1.1$ & $2.14 \mathrm{a} \pm 0.4$ \\
Los Molinos & $3.01 \mathrm{~g} \pm 0.4$ & $1.74 \mathrm{~g} \pm 0.2$ & & $3.11 \mathrm{c} \pm 0.7$ & $1.80 \mathrm{c} \pm 0.1$ \\
Ixtlán de Juárez & $3.05 \mathrm{f} \pm 1.5$ & $1.77 \mathrm{f} \pm 0.1$ & & $2.25 \mathrm{~g} \pm 0.5$ & $1.30 \mathrm{~g} \pm 0.2$ \\
Yudolahuerta & $10.57 \mathrm{a} \pm 1.1$ & $6.13 \mathrm{a} \pm 0.1$ & & $2.50 \mathrm{f} \pm 1.6$ & $1.45 \mathrm{f} \pm 0.2$ \\
Rancho Nuevo & $4.68 \mathrm{c} \pm 0.7$ & $2.71 \mathrm{c} \pm 0.6$ & & $2.66 \mathrm{~d} \pm 0.8$ & $1.54 \mathrm{~d} \pm 0.1$ \\
\hline
\end{tabular}

*Letras diferentes entre columnas de la misma procedencia indican diferencias significativas entre el contenido de acículas, de acuerdo a la prueba de rangos múltiples de Tukey $(\mathrm{p}<0.05)$. El dato después del \pm es el error estándar de la media. 
Ybarra (2009), en un reforestaciones con P. pinceana y $P$. pseudostrobus, reportan una caída de 2.850 y 4.120 Mg C ha ${ }^{-1}$, respectivamente. Por lo que la baja producción de hojarasca tanto en $P$. greggii como en $P$. oaxacana registrada en el presente estudio, comparada con los reportes antes mencionados, podría deberse a la limitada productividad del sitio, como mencionan Domínguez-Calleros et al. (2001), quienes reportan la influencia de las características del sitio y las condiciones climáticas sobre la sobrevivencia, rendimiento y el volumen de madera, para Pinus pseudostrobus Lindl., $P$. greggii Engelm. y $P$. cembroides Zucc.; mientras que Návar-Cháidez y Jurado-Ybarra (2009), mencionan que la productividad de hojarasca y biomasa radicular en comunidades forestales está influenciada por la capacidad productiva de la especie en cuestión.

La acumulación de mantillo (partes muertas de la vegetación, incluyendo hojas, corteza, ramas, acículas, flores, frutos, entre otros) representa una ruta biológica muy importante para la transferencia de elementos inmovilizados en la vegetación al suelo (Yang et al., 2005; Osman, 2013), favoreciendo la recuperación del contenido de materia orgánica del suelo y promoviendo el ciclaje de nutrimentos (Bhat \& Jan, 2010). Osman (2013) menciona que la caída de hojarasca depende de factores como la composición del rodal, la edad de los árboles, el área basal, la densidad, latitud, altitud, estación del año. En Pinus oaxacana Mirov., la edad pudo influir en la caída de hojarasca, ya que la plantación inicio en 1997. También la estación del año en la que se tomaron las muestras de suelo, que fue en marzo de 2013, y el pico de acumulación de acículas en bosques templados es de julio a octubre (Edmonds \& Murray, 2002).

Materia orgánica y Carbono orgánico.

Para la especie Pinus greggii (Tabla 3) la procedencia El Madroño tanto en Tlacotepec Plumas como en Magdalena Zahuatlán presentó alto contenido de MO (7.62\% y 7.12\%, respectivamente). En el contenido de Carbono orgánico, en la comparación entre comunidades, se encontró diferencia estadísticamente significativa $(\mathrm{p}<0.05)$ en ambas comunidades, que como se puede apreciar en la Tabla 3, fue mayor en Tlacotepec Plumas que en Magdalena Zahuatlán.

En un estudio similar, Valencia-Manzo et al. (2006) evaluaron el desarrollo de Pinus greggii en Tlacotepec Plumas y Magdalena Zahuatlán, considerando las mismas procedencias consideradas en este estudio, y mencionan que el suelo de Tlacotepec plumas presentó mayor contenido de materia orgánica (3.0\% y 2.5\%, respectivamente) y de nitrógeno $(0.18 \%$ y $0.10 \%$, respectivamente) que en Magdalena Zahuatlán. Esto concuerda con lo encontrado en este trabajo, ya que en promedio el contenido de MO fue significativamente mayor en Tlacotepec Plumas (7.1592\%) que en Magdalena Zahuatlán (6.3424\%). Esto debido a que la plantación es un área sin aprovechamiento forestal, lo que ha permitido la acumulación de $\mathrm{C}$ y $\mathrm{N}$ bajo el dosel de los árboles de ambas especies.

Para Pinus oaxacana Mirov. (Tabla 4) la procedencia Magdalena Zahuatlán presentó mayor contenido de materia orgánica en Tlacotepec Plumas (6.99 Mg C ha-1), mientras que en Magdalena Zahuatlán fue la procedencia Los Molinos (9.74 Mg C $\left.\mathrm{ha}^{-1}\right)$. Para la especie $P$. oaxacana no se encontraron reportes sobre la cantidad de materia orgánica que se acumula bajo su dosel. En cuanto al contenido de Carbono orgánico entre comunidades se encontró diferencia estadísticamente significativa $\left(\mathrm{p}^{<0.05}\right)$ que, como se puede apreciar en la Tabla 4, fue mayor en la comunidad de Tlacotepec plumas que en Magdalena Zahuatlán. La procedencia que presentó el mayor contenido de CO fue Los Molinos en Tlacotepec Plumas (33.90 Mg C ha-1) y Magdalena Zahuatlán en

Tabla 3. Contenido de materia orgánica (\%) y carbono orgánico (\%) en suelos asociados a Pinus greggii Engelm. en Tlacotepec Plumas y Magdalena Zahuatlán, Oaxaca, a una profundidad de $0-20 \mathrm{~cm}$.

\begin{tabular}{llllll}
\hline \multirow{2}{*}{ Procedencias } & \multicolumn{2}{c}{ Tlacotepec Plumas } & & \multicolumn{2}{c}{ Magdalena Zahuatlán } \\
\cline { 2 - 3 } \cline { 5 - 6 } & \multicolumn{1}{c}{$\% \mathrm{MO}$} & \multicolumn{1}{c}{$\% \mathrm{CO}$} & & \multicolumn{1}{c}{$\% \mathrm{MO}$} & \multicolumn{1}{c}{$\% \mathrm{CO}$} \\
\hline Xichicoatlán & $6.81 \mathrm{i} \pm 0.01$ & $23.73 \mathrm{ab} \pm 0.4$ & & $6.16 \mathrm{fg} \pm 0.004$ & $21.43 \mathrm{~cd} \pm 0.9$ \\
Puerto San Juan & $6.69 \mathrm{j} \pm 0.01$ & $23.27 \mathrm{bc} \pm 1.2$ & & $6.92 \mathrm{e} \pm 0.01$ & $24.08 \mathrm{bc} \pm 0.01$ \\
El Piñón & $5.93 \mathrm{k} \pm 0.02$ & $20.64 \mathrm{c} \pm 0.5$ & & $6.14 \mathrm{~g} \pm 0.002$ & $21.40 \mathrm{~cd} \pm 0.2$ \\
Los Lirios & $7.28 \mathrm{e} \pm 0.002$ & $25.32 \mathrm{ab} \pm 0.7$ & & $6.19 \mathrm{f} \pm 0.005$ & $21.52 \mathrm{~cd} \pm 0.6$ \\
Com. Durango & $7.32 \mathrm{e} \pm 0.01$ & $25.46 \mathrm{ab} \pm 0.6$ & & $6.98 \mathrm{~d} \pm 0.01$ & $24.33 \mathrm{bc} \pm 0.7$ \\
Molango & $7.01 \mathrm{~g} \pm 0.003$ & $24.41 \mathrm{ab} \pm 0.5$ & & $6.99 \mathrm{~d} \pm 0.02$ & $24.35 \mathrm{bc} \pm 0.6$ \\
Pto. Los Conejos & $7.21 \mathrm{f} \pm 0.01$ & $25.11 \mathrm{ab} \pm 0.4$ & & $5.18 \mathrm{j} \pm 0.01$ & $18.02 \mathrm{e} \pm 0.4$ \\
Laguna Atezca & $7.58 \mathrm{bc} \pm 0.01$ & $26.37 \mathrm{a} \pm 0.2$ & & $7.52 \mathrm{~b} \pm 0.004$ & $26.16 \mathrm{ab} \pm 0.6$ \\
El Madroño & $7.62 \mathrm{ab} \pm 0.01$ & $26.50 \mathrm{a} \pm 0.3$ & & $7.12 \mathrm{c} \pm 0.005$ & $27.54 \mathrm{a} \pm 0.6$ \\
Jamé & $7.47 \mathrm{~d} \pm 0.01$ & $26.02 \mathrm{ab} \pm 0.4$ & & $7.91 \mathrm{a} \pm 0.01$ & $24.78 \mathrm{ab} \pm 0.9$ \\
Santa Anita & $6.91 \mathrm{~h} \pm 0.01$ & $24.07 \mathrm{ab} \pm 0.3$ & & $6.00 \mathrm{~h} \pm 0.01$ & $20.95 \mathrm{de} \pm 0.7$ \\
Ej. 18 de marzo & $7.55 \mathrm{c} \pm 0.01$ & $26.25 \mathrm{a} \pm 0.7$ & & $5.35 \mathrm{i} \pm 0.01$ & $18.65 \mathrm{de} \pm 0.2$ \\
Tres lagunas & $7.65 \mathrm{a} \pm 0.01$ & $26.61 \mathrm{a} \pm 0.5$ & & $3.94 \mathrm{k} \pm 0.02$ & $11.71 \mathrm{f} \pm 0.4$ \\
\hline *Letras diferentes entre columnas de la misma procedencia indican diferencias significativas \\
entre el contenido de acículas, de acuerdo a la prueba de rangos múltiples de Tukey $(\mathrm{p}<0.05)$.
\end{tabular}


Tabla 4. Contenido de materia orgánica (\%) y carbono orgánico (\%) en suelos asociados a Pinus oaxacana Mirov. en Tlacotepec Plumas y Magdalena Zahuatlán, Oaxaca, a una profundidad de $0-20 \mathrm{~cm}$.

\begin{tabular}{|c|c|c|c|c|}
\hline \multirow[t]{2}{*}{ Procedencia } & \multicolumn{2}{|c|}{ Tlacotepec Plumas } & \multicolumn{2}{|c|}{ Magdalena Zahuatlán } \\
\hline & \%MO & $\% \mathrm{CO}$ & \%MO & $\% \mathrm{CO}$ \\
\hline Tlacotepec Plumas & $6.43 \mathrm{~d} \pm 0.006$ & $29.17 \mathrm{e} \pm 0.1$ & $8.38 \mathrm{f} \pm 0.002$ & $22.40 c \pm 0.2$ \\
\hline San Miguel Peras & $4.96 \mathrm{~g} \pm 0.018$ & $30.23 c \pm 0.1$ & $8.69 \mathrm{c} \pm 0.002$ & $17.30 \mathrm{e} \pm 0.1$ \\
\hline San Miguel Aloapan & $5.93 f \pm 0.008$ & $32.80 \mathrm{~b} \pm 0.1$ & $9.42 b \pm 0.006$ & $20.66 \mathrm{~d} \pm 0.2$ \\
\hline Magdalena Zahuatlán & $6.99 \mathrm{a} \pm 0.005$ & $30.0 \mathrm{~cd} \pm 0.04$ & $8.61 \mathrm{~d} \pm 0.006$ & $24.35 \mathrm{a} \pm 0.1$ \\
\hline Los Molinos & $6.07 e \pm 0.02$ & $33.90 \mathrm{a} \pm 0.05$ & $9.74 \mathrm{a} \pm 0.004$ & $21.16 \mathrm{~d} \pm 0.1$ \\
\hline Ixtlán de Juárez & $4.69 \mathrm{~h} \pm 0.007$ & $28.56 f \pm 0.2$ & $8.20 \mathrm{~g} \pm 0.006$ & $16.32 \mathrm{f} \pm 0.1$ \\
\hline Yudolahuerta & $6.81 b \pm 0.006$ & $27.73 g \pm 0.1$ & $7.97 \mathrm{~h} \pm 0.004$ & $23.72 \mathrm{ab} \pm 0.1$ \\
\hline Rancho Nuevo & $6.69 \mathrm{c} \pm 0.006$ & 29.65de \pm 0.1 & $8.52 \mathrm{e} \pm 0.01$ & $23.31 \mathrm{~b} \pm 0.1$ \\
\hline
\end{tabular}

Magdalena Zahuatlán (24.35 Mg C ha-1), mientras que San Miguel Peras, que había presentado mayor acumulación de acículas, presentó bajo contenido de materia orgánica.

En los primeros $20 \mathrm{~cm}$ de profundidad del suelo es donde se acumula la mayor actividad microbiana de síntesis y descomposición de materia orgánica (FélixHerrán et al., 2014), de estos procesos se deriva la acumulación de materia orgánica, las acículas secas de pino presentan alto contenido de lignina (25.9330.05\%) (Díaz-Aguirre et al., 2007) y taninos (0.07$0.12 \%)$, precursores de las sustancias húmicas en la materia orgánica, alta relación C/N (alrededor a 150), además presentan bajo contenido de Ca (39.5-54.3\%) y Mg (10.8-18.8\%) (Bernabé-Santiago et al., 2013), por lo que la descomposición sería lenta y al final del proceso de descomposición se tendría un mantillo con alto contenido de materia orgánica (Millar, 2012; FélixHerrán et al., 2014). En el estudio realizado por Monreal et al. (2005) encontraron que en los sistemas con vegetación forestal, el C acumulado en la primera capa del suelo $0-15 \mathrm{~cm}$ fue casi el doble del encontrado en la profundidad 15-30 cm lo que indica que en la primera capa se concentra más el C del suelo.

Aunque la composición química de las acículas es característica para cada especie de coníferas, pero esta varía considerablemente con la edad de la hoja y la estación del año (Napp-Zinn, 1966), por ejemplo en un estudio con Pinus sylvestris L. y Picea abies L., se encontró que los nutrimentos se concentran en las hojas en primavera y se traslocan en verano cuando el crecimiento del árbol es más activo, señalando que los nutrimentos son más estables durante el otoño (Tamm, 1955). En este estudio la plantación inicio en 1997, por lo que la edad de los árboles también podría haber influido en la caída de acículas y en el contenido de materia orgánica final.

En la especie Pinus oaxacana Mirov, la procedencia de Los Molinos presentó el mayor contenido de Carbono total con $33.90 \mathrm{Mg} \mathrm{C}^{-1}$ en la comunidad de Tlacotepec Plumas, mientras que Magdalena Zahuatlán presentó el mayor contenido en
Magdalena Zahuatlán con 24.35 Mg C ha-1. Estos resultados concuerdan con lo reportado por Ordoñez \& Masera (2001) y Ordoñez \& García (2001), quienes evaluaron el contenido de Carbono total en suelos asociados a Pinus pseudostrobus y Pinus oaxacana en la comunidad de Magdalena Zahuatlán, y encontraron que la acumulación de $\mathrm{C}$ en los suelos con $P$. pseudostrobus fue mayor que el $\mathrm{C}$ en suelos con $P$. oaxacana, los autores atribuyeron esto a que $P$. pseudostrobus se asocia con otras arbóreas y arbustivas lo que favorece la acumulación de $\mathrm{C}$ en el suelo, además de factores edáficos y climáticos que influyen para obtener variaciones.

\section{Conclusiones.}

Se presentó variación en cuanto a la cantidad de CO acumulado en las muestras de suelos asociados a Pinus greggii Engelm. y Pinus oaxacana Mirov, esta variación se presentó entre localidades, especies y procedencias.

Con base en los resultados obtenidos en el presente estudio, se puede recomendar el uso Pinus oaxacana Mirov., si la intención de la plantación es la de captar C (bonos verdes) o bien para implementarla en programas de reforestación en suelos perturbados.

\section{Literatura citada.}

Anderegg W. R., Berry J. A., Smith D. D., Sperry J. S., Anderegg L. D. \& Field C. B. 2012. Proc. Natl. Acad. Sci. USA 109: 233-237.

Bernabé-Santiago R., Ávila-Calderón L. E. A. \& RutiagaQuiñones J. G. 2013. Componentes químicos de la madera de cinco especies de pino del municipio de Morelia, Michoacán. Madera y Bosques, 19(2): 21-35.

Bhat N. H. \& Jan S. 2010. Litterfall and Nutrient Return in Ulmus villosa Forests of Dachigam National Park, Jammu and Kashmir. Res J Agric Sci 1(4):363-365.

Castillo M. L. E. 2005. Elementos de muestreo de poblaciones. 2da. Edición, Universidad Autónoma Chapingo, Chapingo, Edo. de México, 267 p.

Denman, G. Brasseur, A. Chidthaisong, P. Ciais, P.M. Cox, R.E. Dickinson, D. Hauglustaine, C. Heinze, E. Holland, D. Jacob, U. Lohmann, S. Ramachandran, P.L. da Silva Dias, S.C. Wofsy \& X. Zhang. 2007. Couplings between 
changes in the climate system and biogeochemistry. Páginas 499 - 587. En: S. Solomon, D. Qin, M. Manning, Z. Chen, M. Marquis, K.B. Averyt, M. Tignor, H.L. Miller (eds.), Climate change 2007: The physical science basis. Contribution of working group 1 to the Fourth Assessment Report of the Intergovernmental Panel on Climate Change. Cambridge University Press, Cambridge, United Kingdom and New York, NY, USA

Díaz-Aguirre S., Alessandrini Díaz M. \& Herrera García A. 2007. Comportamiento del follaje de Pinus caribaea var. Caribaea y Pinus tropicalis en el desarrollo de una metodología para la obtención de cera conífera, pasta clorofila-caroteno y residuo forrajero a escala de banco. Revista Cubana de Química, 19(1): 81-83.

Domínguez-Calleros P. A., J. J. Návar-Cháidez \& J. A. Loera-Ortíz. 2001. Comparación del rendimiento de pinos en la reforestación de sitios marginales en Nuevo León. Madera y Bosques, 7(1): 27-35.

Edmonds R. L. \& Murray G. L. D. 2002. Overstory litter inputs and nutrient returns in an old-growth temperate forest ecosystem, Olympic National Park, Washington. Can J For Res 32(4):742-750.

FAO. 2006. Global forest resource assessment 2005, Main Report, Forestry Paper 147, FAO, Rome.

Félix-Herrán J. A., García-Gutiérrez C. \& ArmentaBojórquez A. D. 2014. Caracterización fisicoquímica y orgánica de suelos y abonos orgánicos. Páginas $11-71$. En: Técnicas de caracterización de suelos y abonos orgánicos, ISBN: 978-607-8347-34-6, editado por Fundación Produce Sinaloa A. C.

Gorte R. W. \& Sheikh P. A. 2010. Deforestation and climate change. CRC Report for Congress.

Gutiérrez-Vázquez, M. H., Méndez-González J., FloresLópez C., Ramírez-Díaz J. A. \& B. N. Gutiérrez-Vázquez B. N. 2012. Caída de hojarasca en plantaciones de Pinus greggii Engelm. y Pinus cembroides Zucc., en Coahuila, México. Rev. Fitotec. Mex. 35 (2): 129-132

IPCC, 2014: Annex II: Glossary [Mach, K.J., S. Planton and C. von Stechow (eds.)]. In: Climate Change 2014: Synthesis Report. Contribution of Working Groups I, II and III to the Fifth Assessment Report of the Intergovernmental Panel on Climate Change [Core Writing Team, R.K. Pachauri and L.A. Meyer (eds.)]. IPCC, Geneva, Switzerland, pp. 117-130.

López O., J. 2012. Carbono orgánico e inorgánico en el suelo en plantaciones de dos especies de pino (Pinus oaxacana Mirov y Pinus greggii Engelm.) de diferentes edades en Magdalena Zahuatlán, Oaxaca. Tesis Profesional ITSMIGRA. 34 p.

Millar C. S. 2012. Decomposition of coniferous leaf litter. Páginas 105-127. En: Biology of plant litter decomposition, Volume 1. C. H. Dickinson (ed.), Edited by Elsevier. U.S.

Napp-Zinn K. 1966. Anatomie des Blattes. I. Gymnospermen. Handbuch der Pflanzenanatomie VII. Gebrüder Borntraeger, Berlin.

Návar-Cháidez J.J. \& Jurado-Ybarra E. 2009. Productividad foliar y radicular en ecosistemas forestales del noreste de México. Rev. Ciencia Forestal en México. 34(106): 89 106.

NOM-021-RECNAT-2000. Método AS-07 para determinar contenido de materia orgánica en muestras de suelo. Aprobada por el Comité Consultivo Nacional de Normalización para la Conservación, Protección,
Restauración y Aprovechamiento de los Recursos Forestales de Suelos y Costas, en sesión celebrada el 14 de agosto de $2001.85 \mathrm{p}$.

Ordoñez S. N. \& O. M. García O. M. 2001. Almacenamiento de Carbono en un bosque de P. pseudostrobus en Nuevo san Juan Michoacán. Madera y bosques. Xalapa, México. pp. 27-47.

Ordóñez, J. A. \& Masera O. 2001. Captura de Carbono ante el cambio climático. Madera y Bosques. 7(1): 3-12

Osman K. T. 2013. Forest soils properties and management. Edited by Springer International Publishing, New Delhi, India, p. 217.

Pérez C. A., Goya J. F., Bianchini F., Frangi J. L. \& Fernández R. 2006. Productividad aérea y ciclo de nutrientes en plantaciones de Pinus taeda L. en el norte de la provincia de Misiones, Argentina. Revista Interciencia, 31(11): $794-801$

Pribyl D. W. 2010. A critical review of the conventional SOC to SOM conversion factor. Geoderma 156:75-83.

Price S. P., Bradford M. A. \& Ashton M. S. 2012. Characterizing organic carbon stocks and flows in forest soils. Páginas 233 - 244. En: Ashton MS et al. (eds) Managing forest carbon in a changing climate. Springer Science + Business Media BV

SAS System for Windows. 2002. By SAS Institute Inc., Cary, NC, USA.

Secretaria de Gobernación (SEGOB). 2014. Programa Nacional Forestal 2014-2018. Disponible en: http://www.dof.gob.mx/nota_detalle.php?codigo $=53424$ 98\&fecha $=28 / 04 / 2014$

Secretaria de Medio Ambiente y Recursos Naturales (SEMARNAT). 2007. ¿Y el medio ambiente? Problemas en México y el mundo. SEMARNAT. México. 192 p.

Smith P., M. Bustamante, H. Ahammad, H. Clark, H. Dong, E.A. Elsiddig, H. Haberl, R. Harper, J. House, M. Jafari, O. Masera, C. Mbow, N.H. Ravindranath, C.W. Rice, C. Robledo Abad, A. Romanovskaya, F. Sperling, \& F. Tubiello, 2014: Agriculture, Forestry and Other Land Use (AFOLU). Páginas 811 - 922. En: Climate Change 2014: Mitigation of Climate Change. Contribution of Working Group III to the Fifth Assessment Report of the Intergovernmental Panel on Climate Change [Edenhofer, O., R. Pichs-Madruga, Y. Sokona, E. Farahani, S. Kadner, K. Seyboth, A. Adler, I. Baum, S. Brunner, P. Eickemeier, B. Kriemann, J. Savolainen, S. Schlömer, C. von Stechow, T. Zwickel and J.C. Minx (eds.)]. Cambridge University Press, Cambridge, United Kingdom and New York, NY, USA.

Soil Survey Staff. 2014. Soil Survey Field and Laboratory Methods Manual. Soil Survey Investigations Report No. 51, Version 2.0. R. Burt and Soil Survey Staff (ed.). U.S. Department of Agriculture, Natural Resources Conservation Service, p. 487.

Tamm C. O. 1955. Studies on forest nutrition: Seasonal variation in the nutrient content of conifer needles. Medd. Statens Skogsforskningsinst. 45(5/6): 5-34.

Valencia-Manzo, S., Velasco-García M. V., GómezCárdenas M., Ruiz Muñoz M. \& Capó Arteaga M. A. 2006. Ensayo de procedencias de Pinus greggii Engelm. en dos localidades de la mixteca alta de Oaxaca, México Revista Fitotecnia Mexicana, 29(1): 27-32.

Yang W.Q., Wang K. Y., Kellomaki S. \& Gong H. 2005. Litter dynamics of three subalpine forests in Western Sichuan. Pedosphere. 15(5):653-659. 
${ }^{1}$ Estudiante de Ingeniería Forestal, Universidad Autónoma Indígena de México.

${ }^{2}$ Campo Experimental Valles Centrales de Oaxaca, Melchor Ocampo No. 7, INIFAP.

${ }^{3}$ Investigador Titular del Centro Interdisciplinario de Investigación para el Desarrollo Integral Regional del Instituto Politécnico Nacional (CIIDIR-IPN).

${ }^{4}$ Profesor de Ingeniería Forestal, Universidad Autónoma Indígena de México. Correo-e: jfelixherran@hotmail.com (autor para correspondencia). 